\title{
Distinctive Features of Saccadic Intrusions and Microsaccades in Progressive Supranuclear Palsy
}

\author{
Jorge Otero-Millan, ${ }^{1,5}$ Alessandro Serra, ${ }^{2,3}$ R. John Leigh, ${ }^{2}$ Xoana G. Troncoso, ${ }^{1,4}$ Stephen L. Macknik, ${ }^{1}$ \\ and Susana Martinez-Conde ${ }^{1}$ \\ ${ }^{1}$ Barrow Neurological Institute, Phoenix, Arizona 85013, ${ }^{2}$ Veterans Affairs Medical Center, Case Western Reserve University, Cleveland, Ohio 44106, \\ ${ }^{3}$ Institute of Clinical Neurology, University of Sassari, 07100 Sassari, Italy, ${ }^{4}$ California Institute of Technology, Pasadena, California 91125, and ${ }^{5}$ University \\ of Vigo, 36310 Vigo, Spain
}

The eyes do not stay perfectly still during attempted fixation; fixational eye movements and saccadic intrusions (SIs) continuously change the position of gaze. The most common type of SI, square-wave jerks (SWJs), consists of saccade pairs that appear purely horizontal on clinical inspection: the first saccade moves the eye away from the fixation target, and after a short interval, the second saccade brings it back toward the target. SWJs are prevalent in certain neurological disorders, including progressive supranuclear palsy (PSP). Here, we developed an objective method to identify SWJs. We found that SWJs are more frequent, larger, and more markedly horizontal in PSP patients than in healthy human subjects. Furthermore, the loss of a vertical component in fixational saccades and SWJs was the eye movement feature that best distinguished PSP patients from controls. We moreover determined that, in PSP patients and controls, the larger the saccade the more likely it was part of a SWJ. Furthermore, saccades produced by PSP patients had equivalent properties whether they were part of a SWJ or not, suggesting that normal fixational saccades (microsaccades) are rare in PSP. We propose that fixational saccades and SIs are generated by the same neural circuit and that, both in PSP patients and in controls, SWJs result from a coupling mechanism that generates a second corrective saccade shortly after a large fixation saccade. Because of brainstem and/or cerebellum impairment, fixational saccades in PSP are abnormally large and thus more likely to trigger a corrective saccade, giving rise to SWJs.

\section{Introduction}

During visual fixation of a stationary target, the eyes are in constant motion: fixational eye movements (including microsaccades, drift, and tremor) continually change the position of gaze (Martinez-Conde et al., 2004, 2009).

In addition, most subjects show saccadic intrusions (SIs), predominantly horizontal saccades that "intrude on" or interrupt accurate fixation. Square-wave jerks (SWJs) are the most common type of SI, with a typical frequency of one SWJ per $3 \mathrm{~s}$ in healthy subjects (Sharpe and Fletcher, 1986; Abadi and Gowen, 2004). Each SWJ consists of a small, conjugate, mainly horizontal saccade that moves the eye away from the fixation target, followed after a short interval by a corrective saccade that returns the eye toward the target (see Fig. 1; supplemental Movie 1, available at www.jneurosci.org as supplemental material).

Received May 17, 2010; revised Jan. 13, 2011; accepted Jan. 20, 2011.

This work was supported by the following funding agencies: Barrow Neurological Foundation (S.L.M., S.M.-C.) Arizona Biomedical Research Commission (S.M.-C.), National Science Foundation Awards 0643306 and 0852636 (S.M.-C.), National Institutes of Health Grant EY06717 (R.J.L.), the Office of Research and Development, Medical Research Service, Department of Veterans Affairs (R.J.L.), The Evenor Armington Fund (R.J.L.), and the Oasi Institute for Research and Care (Instituto di Ricovero e Cura a Carattere Scientifico) on Mental Retardation and Brain Aging (Troina, Italy) (A.S.). J.0.-M. is a Fellow of the Pedro Barrié de la Maza Foundation. We thank Isabel Gomez-Caraballo, Manuel Ledo, and Andrew Danielson for technical assistance, and Hector Rieiro for his comments.

Correspondence should be addressed to Dr. Susana Martinez-Conde, Laboratory of Visual Neuroscience, Division of Neurobiology, Barrow Neurological Institute, 350 West Thomas Road, Phoenix, AZ 85013. E-mail: smart@neuralcorrelate.com.

DOI:10.1523/JNEUROSCI.2600-10.2011

Copyright $\odot 2011$ the authors $\quad 0270-6474 / 11 / 314379-09 \$ 15.00 / 0$
Frequent SWJs are characteristic of some neurological diseases, for instance Friedrich's ataxia and parkinsonian disorders such as progressive supranuclear palsy (PSP) (Troost and Daroff, 1977; Leigh and Zee, 2006; Fahey et al., 2008). Thus, SWJs are of diagnostic interest to the neurologist (supplemental Movie 2, available at www.jneurosci.org as supplemental material).

Microsaccades and SWJs have been considered different oculomotor phenomena because of some seemingly dissimilar features. For instance, microsaccades tend to be smaller than SWJs and often display an oblique trajectory, whereas SWJs appear to be purely horizontal on clinical inspection (SWJ studies have primarily focused on neurological patients, whereas microsaccade research is mostly constrained to healthy subjects). Despite these apparent distinctions, minimally enlarged microsaccades (beyond 15-30 arcmin) tend to produce square-wave coupling in healthy subjects (Ditchburn and Ginsborg, 1953; Vedel-Jensen, 1966; Yamazaki, 1968; Feldon and Langston, 1977). SWJs and microsaccades have other important similarities. For example, they both follow the saccadic peak velocity/magnitude relationship and are affected by attention (Hafed and Clark, 2002; Gowen et al., 2005). Gowen et al. (2007) suggested that "SI and microsaccades may lie on a continuum of fixational instabilities" and "may [be] different names given to the same type of fixational eye movement," proposing that future studies investigate whether both microsaccades and SIs display a correlation between size and coupling. If present, microsaccadic coupling would follow from current models of microsaccade generation (Rolfs et al., 
2008b; Hafed et al., 2009) (i.e., the visual error created by an initial large microsaccade could trigger a corrective saccade in the opposite direction). Here, we present the results of such an examination.

From here on and for the sake of clarity, we will refer to all saccades made during attempted fixation as fixational saccades or simply saccades.

We needed to develop an objective algorithm to characterize SWJs in healthy controls and PSP patients, and to distinguish saccades in SWJs from other involuntary fixational saccades. Our primary goal was to identify any differences between saccades and SWJs made by PSP patients versus those made by healthy subjects. A secondary goal was to test the hypothesis that microsaccades and SIs lie on a continuum [if so, it would suggest that microsaccades and SI have a common oculomotor substrate (Gowen et al., 2007)]. We focused on PSP because it causes frequent, large SWJs as well as a predominantly vertical saccadic palsy.

\section{Materials and Methods}

Subjects. We tested three subject groups: the PSP group comprised 10 patients ( 5 females; age range, 58-74; median, 66.5 years), diagnosed as probable PSP according to the criteria of the National Institute of Neurological Disorders and Stroke-Society for PSP study (Litvan et al., 1996); duration of disease was 3-7 years, and median, 4 . Their clinical features are summarized in supplemental Table 1 (available at www. jneurosci.org as supplemental material). The first group of control subjects ("older control group") comprised seven age-matched healthy subjects (one female; age range, 58-74; median, 65 years); all had corrected visual acuity better than $20 / 30$. The second group of control subjects ("younger control group") comprised seven healthy subjects (five females; age range, 22-38 years; median, 31 years) with normal or corrected-to-normal vision.

The PSP group and the older control group were tested at the Veterans Affairs Medical Center, Case Western Reserve University (Cleveland $\mathrm{OH})$; each subject participated in one experimental session lasting $\sim 30$ $\mathrm{min}$. The younger control group was tested during a previously reported study [Otero-Millan et al. (2008), their "blank scene" fixation condition] at the Barrow Neurological Institute (Phoenix, AZ); each subject participated in three experimental sessions, of $\sim 60 \mathrm{~min}$.

All subjects were naive to the purpose of the experiments and gave written informed consent in accord with our institutional review boards and the Declaration of Helsinki.

Eye-tracking apparatus. For the subjects studied in Cleveland (PSP group and older control group), monocular or binocular eye movements were obtained using the magnetic field/search coil technique (Robinson, 1963); search coils were calibrated on a protractor device before each experimental session. Subjects sat in a vestibular chair with their heads stabilized by a chair-fixed restraint. Coil signals were low-pass filtered (bandwidth, $0-150 \mathrm{~Hz}$ ) before digitization at $500 \mathrm{~Hz}$ with 16 bit precision, as previously described (Ramat et al., 1999). The SD of the noise of the coil system was $0.02^{\circ}$.

One should note that, although the magnetic field/search coil technique is precise, it is not necessarily accurate: absolute position depends on the subjects directing their foveal line of sight at the target. Unfortunately, most PSP patients cannot reliably point their foveal line of sight because of their defect in voluntary gaze. Thus, absolute eye position measurements that depend on visually driven eye movements for calibration are inherently unreliable in patients with PSP. For most experimental purposes, however, it is possible to determine the coil signal corresponding approximately to zero eye position in PSP patients, as it predominates even in individuals showing frequent SWJs.

Subjects studied in Phoenix (younger control group) rested their head on a chin rest while their eye position was acquired noninvasively with a fast video-based eye movement monitor (EyeLink II; SR Research). The EyeLink II system records eye movements simultaneously in both eyes (temporal resolution, 500 samples/s; instrument noise, $0.01^{\circ}$ root mean square), in its off-the-shelf configuration.
Video-tracking techniques are generally less precise and accurate than the magnetic field/search coil technique. Thus, absolute eye position measurements were less reliable for the younger control group than for the older control group.

Despite the stated limitations for measuring absolute eye position, both magnetic/search coil and video-tracking techniques reliably detect small saccades (because the detection methods depend on relative changes in eye position, rather than on the absolute position of the eye).

Having two control groups with different subject ages and eyetracking methodology was advantageous, as it suggested that the results from control subjects were not likely specific to either the subjects' age or the recording system used (for details, see Results).

Experimental design. All subjects were asked to maintain gaze fixation during the recordings (minimum fixation duration, $10 \mathrm{~s}$; maximum fixation duration, $120 \mathrm{~s}$ ). In Cleveland (older control group and PSP group), subjects viewed a small target (laser spot subtending $0.1^{\circ}$ ) placed at central position on a tangent screen at $1.4 \mathrm{~m}$ in an otherwise dark room. Verbal encouragement was provided to subjects to sustain steady fixation of the small target during the test period, allowing occasional blinks. In Phoenix (younger control group), subjects fixated a red cross $\left(0.75^{\circ}\right.$ wide $)$ within a $2 \times 2^{\circ}$ window, on a $50 \%$ gray background. This window size produced loose fixation, typical of natural fixation behavior (Martinez-Conde et al., 2000, 2002; Martinez-Conde, 2006). The cross was presented on the center of a linearized video monitor, at $57 \mathrm{~cm}$. The subjects received auditory feedback (a short beep) whenever their gaze left the fixation window for $>500 \mathrm{~ms}(<500 \mathrm{~ms}$ gaze excursions were permitted to allow for blinks). Eye movements exceeding the fixation window were also recorded.

Objective saccade characterization. We identified saccades automatically with an objective detection algorithm (for details, see Engbert and Kliegl, 2003). In those subjects in whom eye position was recorded binocularly, we reduced the amount of potential noise (Engbert, 2006) by considering only binocular saccades, that is, saccades with a minimum overlap of one data sample in both eyes (Laubrock et al., 2005; Engbert, 2006; Engbert and Mergenthaler, 2006; Rolfs et al., 2006; Otero-Millan et al., 2008; Troncoso et al., 2008a,b).

Some saccades are followed by a fast small saccadic, oppositely directed, eye movement called dynamic overshoot, which is often more prominent for the eye that moves in the abducting direction (Kapoula et al., 1986). Unlike in SWJs, a dynamic overshoot follows a saccade without latency between the two movements. We identified dynamic overshoots as saccades that occurred $<20 \mathrm{~ms}$ after a preceding saccade (Møller et al., 2002; Otero-Millan et al., 2008; Troncoso et al., 2008a,b) and considered them part of the preceding saccade (i.e., we did not regard them as new saccades). That is, we modified the end point of the previous saccade to include the overshoot.

Development of an objective algorithm for the automatic detection of SWJs. We defined a SWJ as the combination of one small saccade that moves the eye away from the fixation target, followed after a short period by a second corrective saccade directed back toward the target (Abadi and Gowen, 2004; Leigh and Zee, 2006; Martinez-Conde, 2006) (Fig. 1). To characterize SWJs in an objective manner, we first identified all individual saccades up to $5^{\circ}$ (Engbert and Kliegl, 2003) (see above, Objective saccade characterization). We chose this $5^{\circ}$ upper magnitude threshold to include the range of SWJ magnitudes reported by Abadi and Gowen (2004) $\left(0.1-4.1^{\circ}\right)$ in healthy subjects, and to also allow for potentially larger SWJs magnitudes in PSP patients.

SWJs have three defining characteristics: (1) the two saccades have (approximately) opposite directions, (2) both saccades have similar magnitudes, and (3) the two saccades are separated by a short interval. We developed an objective algorithm to establish, automatically, whether a pair of consecutive saccades constituted a SWJ or not. To do this, we created a SWJ index that measured how similar a given saccade pair was to an "ideal SWJ." The SWJ index combined the three defining characteristics of SWJs described above: (1) the direction dissimilarity of first and second saccade, (2) the magnitude similarity of first and second saccade, and (3) the temporal proximity of first and second saccade, in a single, continuous variable for each saccade pair. If the SWJ index of a 


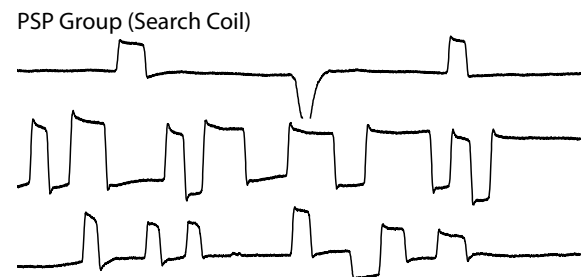

Older Control Group (Search Coil)

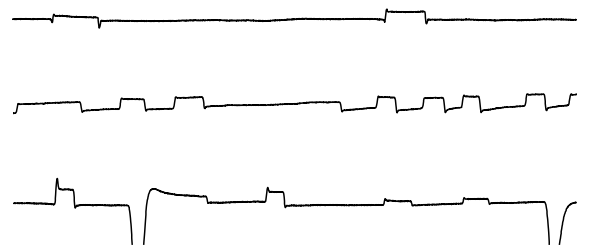

Younger Control Group (Video Tracking)

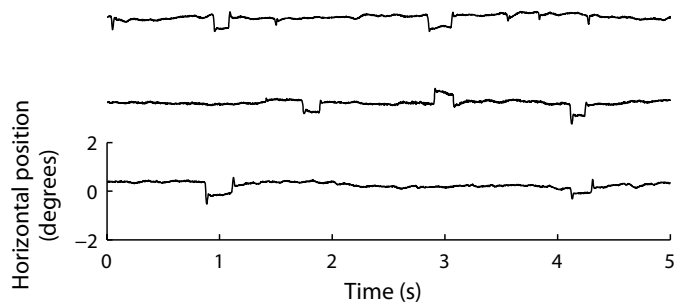

Figure 1. Examples of SWJs in control subjects and PSP patients. SWJs are present in healthy subjects and PSP patients, although they are larger and more prevalent in PSP patients. Each trace represents a 5 s recording of horizontal eye positions containing SWJs. Top, Examples from three PSP patients (recorded with a search coil). Bottom, Examples from six healthy subjects (the top 3 traces were recorded with a search coil; the bottom 3 traces were recorded with a video-based eye-tracking system). Horizontal position and timescales for all traces are as in bottom trace. Notice that the quality of the recordings was comparable for both control groups, despite the use of different eye-tracking methods.

saccade pair was larger than a given threshold, we classified the pair as a potential SWJ.

For a pair $i$ of consecutive saccades, we calculated the direction dissimilarity between saccade 1 and saccade $2, D_{i}=$ direction $_{i 1}-$ direction $_{i 2}$ (Fig. $2 A$ ), the magnitude similarity between the two saccades, $M_{i}=$ $\left(\right.$ magnitude $_{i 1}-$ magnitude $\left._{i 2}\right) /\left(\right.$ magnitude $_{i 1}+$ magnitude $\left._{i 2}\right)\left(\right.$ Fig. $\left._{2} B\right)$, and the temporal proximity or interval between the two saccades, $I_{i}=$ end $_{i 1}-$ start $_{i 2}$ (Fig. 2C).

We defined the SWJ index for the saccade pair $i$ as follows: SWJindex ${ }_{i}=$ $f_{D}\left(D_{i}\right) * f_{M}\left(M_{i}\right) * f_{I}\left(I_{i}\right)$. The functions $f_{D}, f_{M}$, and $f_{I}$ measure how similar $D_{i}, M_{i}$, and $I_{i}$ are to those in an ideal SWJ, and can be thought of as a measure of the probability of a SWJ with characteristics $D_{i}, M_{i}$, and $I_{i}$ (i.e., the most likely SWJs are those closer to the ideal SWJ). We fitted the probability distributions of $D, A$, and $I$ with common distributions (i.e., Gaussian mixture and ex-Gaussian) to estimate the parameters of $f_{D}, f_{M}$, and $f_{I}$. We calculated these distributions using data from pairs of consecutive saccades from the PSP group. SWJs in the PSP group were very prevalent and exhibited large magnitudes, which facilitated their characterization. Indeed, SWJ index was higher on average in the PSP group than in the control groups (Fig. 2G-I). The direction dissimilarity distribution was bimodal (Fig. $2 \mathrm{D}$ ), so we could discard all pairs of consecutive saccades with approximately the same direction. To calculate the fits of the distributions, we used only saccade pairs in which the directions of first and second saccade differed substantially (i.e., between 90 and $270^{\circ}$ ). All the fits were calculated using maximum-likelihood estimation.

We fitted the direction dissimilarity distribution with a mixture of two Gaussians (Fig. 2D). This resulted in a mixture of two Gaussians with almost the same mean $\left(180^{\circ}\right)$ but different SDs, corresponding to the tails $\left(30^{\circ}\right)$ and to the central part of the distribution $\left(7^{\circ}\right)$ and with relative weights 0.4 and 0.6 . If $F_{D}(D)$ is the distribution function given by the fit, and $D_{\text {ideal swJ }}=180^{\circ}$ is the direction difference in an ideal SWJ, we defined the following:

$$
f_{D}\left(D_{i}\right)=\left\{\begin{array}{c}
1-F_{D}\left(D_{i}\right) \quad \text { if } D_{i} \geq D_{\text {ideal SwJ }} \\
F_{D}\left(D_{i}\right) \text { if } D_{i}>D_{\text {ideal SwJ }}
\end{array} .\right.
$$

We fitted the magnitude similarity distribution with a mixture of two Gaussians (Fig. 2E). This resulted in a mixture of two Gaussians with almost the same mean (0) but different SDs, corresponding to the tails $(0.39)$ and to the central part of the distribution (0.16), and with relative weights 0.4 and 0.6 . If $F_{M}(M)$ is the distribution function given by the fit, and $M_{\text {ideal SWJ }}=0$ is the magnitude similarity index in an ideal SWJ, we defined the following:

$$
f_{M}\left(M_{i}\right)=\left\{\begin{array}{c}
1-F_{M}\left(M_{i}\right) \quad \text { if } M_{i} \geq M_{\text {ideal swJ }} \\
F_{M}\left(M_{i}\right) \text { if } M_{i}>M_{\text {ideal swJ }}
\end{array} .\right.
$$

We fitted the intersaccadic interval (ISI) distribution with an exGaussian function (Otero-Millan et al., 2008) (Fig. 2F). This resulted in an ex-Gaussian distribution $F_{I}$ with mean, $120 \mathrm{~ms}$; SD, $60 \mathrm{~ms}$; and $\tau, 180$ ms. If $F_{I}(I)$ is the distribution function given by the fit, and $I_{\text {ideal swJ }}=$ 200 is the ISI in an ideal SWJ, we defined the following:

$$
f_{I}\left(I_{i}\right)=\left\{\begin{array}{c}
1-F_{I}\left(I_{i}\right) \quad \text { if } I_{i} \geq I_{\text {ideal SWJ }} \\
F_{I}\left(I_{i}\right) \quad \text { if } I_{i}>I_{\text {ideal SWJ }}
\end{array}\right.
$$

Finally, we determined the threshold that would establish whether the SWJ index of a given saccade pair corresponded to that of a potential SWJ. We calculated the SWJ index of the same saccade pairs we used to estimate the fits. Then, we used a $k$-means algorithm to cluster all the pairs in two groups, one with high SWJ indices and one with low SWJ indices. Our SWJ index threshold was the boundary between the two groups, in this case, 0.0014 .

Thus, we were able to identify the saccade pairs that met the SWJ criterion. However, it was possible for a single saccade to be part of two different SWJs (that is, a given saccade could be both the second saccade of a given SWJ and the first saccade of the next SWJ), as it might occur with a series of "SWJ oscillations" (Leigh and Zee, 2006). To avoid this possibility, we identified all the sequences of consecutive SWJs that were connected by shared saccades and set up a rule that assigned each saccade to a unique SWJ, as follows: (1) we assigned the first two saccades to the first SWJ, the next two saccades to the second SWJ, etc., or (2) we skipped the first saccade and assigned the next two saccades to the first SWJ, and so on. We applied (1) or (2) to each SWJ sequence as to maximize the total SWJ index. Table 1 summarizes the characteristics of all SWJs automatically detected with our algorithm in each subject group (the population values were consistent with those of individual subjects) (supplemental Table 2, available at www.jneurosci.org as supplemental material).

One should note that the algorithm does not assume a bias in the direction of the SWJs (for instance, there is no presumption that SWJs must be comprised of horizontal saccades). However, the algorithm identifies SWJs that are almost exclusively horizontal in both patients and controls (serving as a validity check of the algorithm, see Results).

The SWJ detection algorithm is freely available for download at http://smc.neuralcorrelate.org/software/swj/.

The methods described allowed us to detect saccades both inside and outside SWJs. By "saccades inside SWJs," or "SWJ saccades," we refer to the two saccades that define a complete SWJ (that is, an initial saccade that moves the eye away from the fixation target, followed by a second, corrective saccade back toward the target). By "saccades outside SWJs," or "non-SWJ saccades," we refer to any other saccades that are not part of SWJs, including fixational microsaccades.

Receiver operating characteristic analysis. We used a receiver operating characteristic (ROC) analysis (Green and Swets, 1966; Swets, 1988; Zweig and Campbell, 1993) to quantify how well different SWJ properties, such as rate, magnitude, and direction, may predict which group a given subject belonged to (PSP patient or healthy control). This analysis makes no assumptions about the underlying distributions (Green and Swets, 1966). To obtain the ROC curve, we plotted the probability of true pos- 
itives as a function of the probability of false positives for all possible criterion levels. The area under the ROC curve provides a measure of the discriminability of two signals and is directly related to the overlap of the two distributions of the property that is being compared (Green and Swets, 1966). In our case, the area under the ROC curve can be interpreted as the probability with which an ideal observer, given a SWJ property from a subject, can correctly determine whether the subject suffers from PSP or not. An area of 0.5 corresponds to completely overlapping distributions (the ideal observer cannot discriminate between the two groups); an area of 1 corresponds to groups that can be perfectly discriminated based on the specific property. To determine whether the value of the area under the curve was better than chance, we calculated significance using a permutation procedure (Green and Swets, 1966; Britten et al., 1992; Hernández et al., 2002; Romo et al., 2004; Feierstein et al., 2006), using $n=1000$ shuffles and a criterion $p<$ 0.01 . We also calculated the "optimal working point" for each of the SWJ properties considered. The optimal working point is the threshold that minimizes the ideal observer's errors (simplified case in which costs of errors and prevalence of disease are not considered). It corresponds to the point in the ROC curve where a line with slope 1 touches the curve (Zweig and Campbell, 1993).

\section{Results}

\section{Comparison of SWJ saccades and non-SWJ saccades}

A SWJ consists of two saccades of opposing directions. We compared the dynamic properties of saccades in SWJs to those of other fixational saccades (up to $5^{\circ}$ ) that were not part of SWJs, in PSP patients and in healthy participants (Fig. 3). SWJ saccades followed the same peak velocity/ magnitude relationship as non-SWJ saccades in all subject groups. However, the slopes of the linear fits were lower (both for SWJ and non-SWJ saccades) in the PSP group than in the control groups (Fig. 3, first row). [The saccadic duration/ magnitude relationships (Evinger et al., 1984; Gruart et al., 1995) are plotted in supplemental Figure 1 (available at www.jneurosci.org as supplemental material).] This indicated that all saccades (both inside and outside SWJs) are slower overall in PSP patients than in healthy participants.

Saccade magnitudes (both inside and outside SWJs) were larger in the PSP group than in the control groups (Fig. 3, second row).

Saccades in SWJs were predominantly horizontal in all three subject groups. Small saccades with vertical components (outside SWJs) were observed in the control groups, but not in the PSP group (Fig. 3, third row), consistent with the vertical saccadic palsy that is a feature of PSP. Since these analyses produced equivalent results in both control groups, despite differences in subjects' age and eye-tracking methodology, we considered all control subjects together for the remainder of the study.
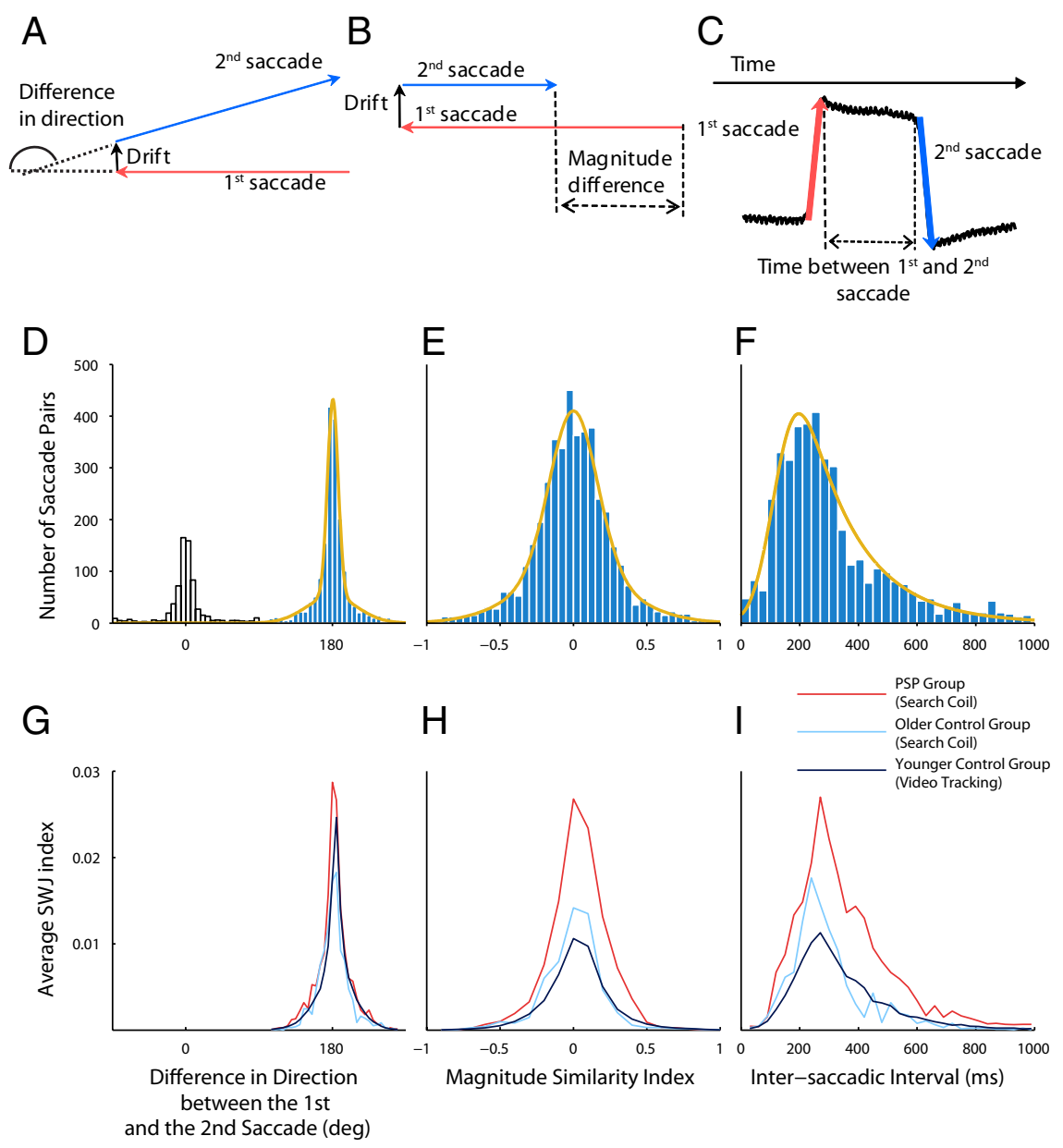

Figure 2. Objective SWJ characterization. We calculated an index that combined the following defining characteristics of SWJs: (1) the first and second saccades should have (approximately) opposite directions, (2) both saccades should have similar magnitudes, and (3) the two saccades should be separated by a short interval. Top row, SWJ characteristics. $\boldsymbol{A}$, The direction dissimilarity of first and second saccades. $\boldsymbol{B}$, The magnitude similarity of first and second saccades. $\boldsymbol{C}$, The temporal proximity of first and second saccades. Middle row, Distributions of SWJ characteristics for saccade pairs in PSP patients and fits used to calculate the SWJ index. presents the fit of the Gaussian mix distribution. The empty bars show the distribution of saccades not used to calculate the fits.

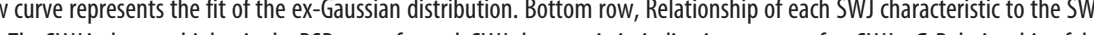
index (for saccade pairs potentially forming SWJS) to the SWJ index. I, Relationship of the temporal proximity or ISI (for saccades pairs potentially forming SWJS) to the SWJ index.

The above findings (i.e., that all saccades in PSP patients follow the peak velocity/magnitude relationship but are slower, larger, and more horizontal than in controls) could indicate that all saccades in PSP are affected in a similar fashion and that PSP patients do not produce regular fixational saccades (i.e., microsaccades). To address this, we compared the magnitude, peak velocity, and vertical component of SWJ saccades to those of non-SWJ saccades in PSP patients and found no significant differences (Fig. $4 A-C$, red bars). In the case of the control subjects, SWJ saccades were significantly different from non-SWJ saccades in all three properties (Fig. $4 A-C$, blue bars).

We also compared the ISIs between the two saccades forming a SWJ (intra-SWJ ISIs) and between consecutive saccades not forming a SWJ (inter-SWJ ISIs) in patients and controls (Fig. $4 D)$. Both patients and controls presented shorter intra-SWJ 
Table 1. Characteristics of SWJs

\begin{tabular}{|c|c|c|c|c|c|c|c|c|c|}
\hline & \multicolumn{3}{|l|}{ SWJ saccades } & \multicolumn{3}{|c|}{ Non-SWJ saccades } & \multicolumn{3}{|l|}{ All saccades } \\
\hline & Controls & PSPs & $p$ value & Controls & PSPs & $p$ value & Controls & PSPs & $p$ value \\
\hline Rate $(\mathrm{N} / \mathrm{s})$ & $0.2 \pm 0.1$ & $0.8 \pm 0.1$ & $<0.0001$ & $0.7 \pm 0.1$ & $0.6 \pm 0.1$ & $>0.01$ & $1.1 \pm 0.2$ & $2.1 \pm 0.2$ & $<0.001$ \\
\hline Number of SWJs & $400 \pm 200$ & $90 \pm 20$ & - & $2000 \pm 1000$ & $300 \pm 100$ & - & $2000 \pm 1000$ & $300 \pm 100$ & - \\
\hline Magnitude (deg) & $0.6 \pm 0.1$ & $1.4 \pm 0.2$ & $<0.001$ & $0.42 \pm 0.04$ & $1.3 \pm 0.2$ & $<0.0001$ & $0.46 \pm 0.04$ & $1.4 \pm 0.2$ & $<0.0001$ \\
\hline Peak velocity (deg/s) & $47 \pm 4$ & $70 \pm 10$ & $<0.01$ & $36 \pm 3$ & $70 \pm 10$ & $<0.001$ & $39 \pm 3$ & $70 \pm 10$ & $<0.001$ \\
\hline Vertical comp (normalized) & $0.37 \pm 0.04$ & $0.14 \pm 0.02$ & $<0.0001$ & $0.59 \pm 0.03$ & $0.20 \pm 0.04$ & $<0.000001$ & $0.52 \pm 0.04$ & $0.16 \pm 0.03$ & $<0.00001$ \\
\hline Intra-SWJ ISI (ms) & $290 \pm 10$ & $280 \pm 10$ & $>0.01$ & & & & & & \\
\hline Inter-SWJ ISI (ms) & $1100 \pm 200$ & $500 \pm 100$ & $<0.001$ & & & & & & \\
\hline SWJ SD of direction difference & $21 \pm 1$ & $9 \pm 2$ & $<0.00001$ & & & & & & \\
\hline Percentage of saccades in SWJs & $30 \pm 10$ & $70 \pm 10$ & $<0.001$ & & & & & & \\
\hline
\end{tabular}

Averages and intersubject SEMs are indicated for each parameter tested. Values of $p$ were determined by $t$ test. To calculate the vertical component of saccades of different sizes, we first normalized the magnitude of all saccades to $1^{\circ}$.
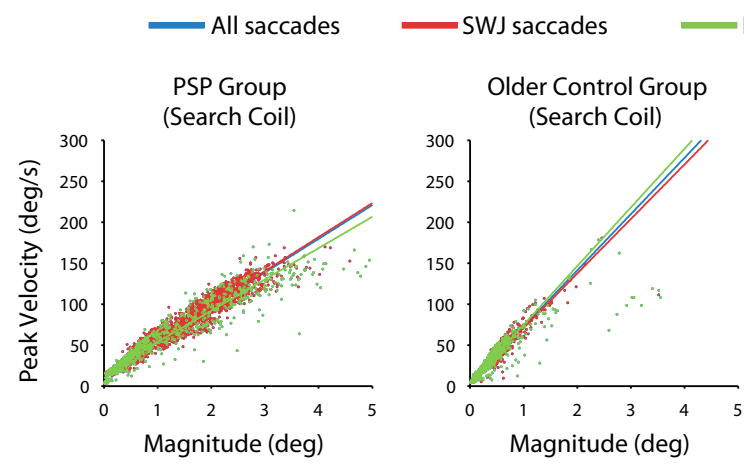

Non-SWJ saccades
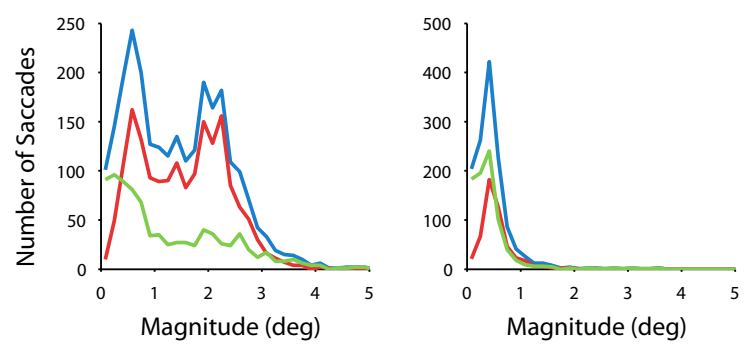

Younger Control Group
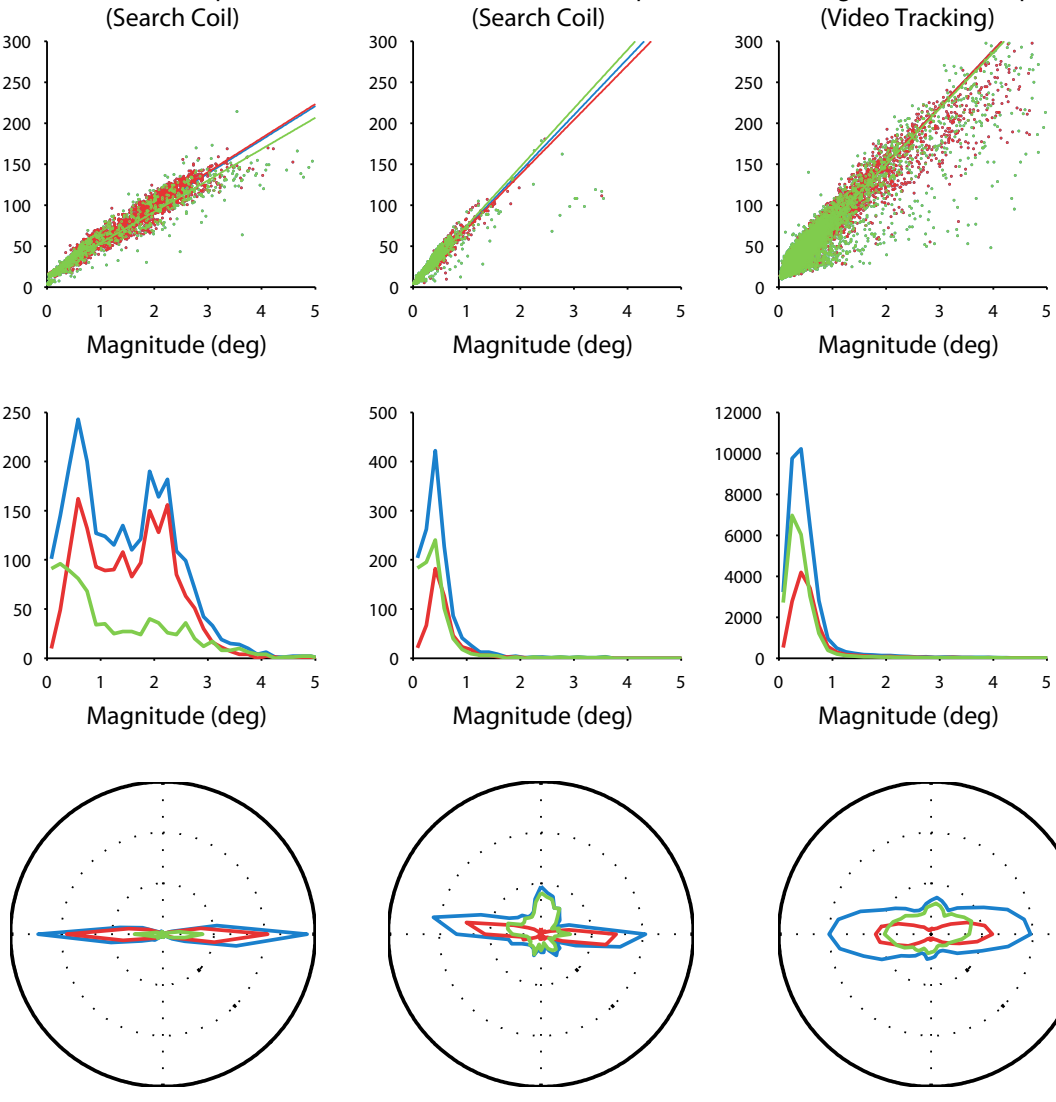

Direction

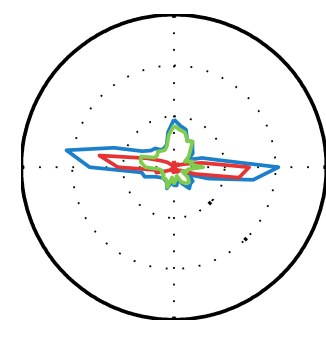

Direction

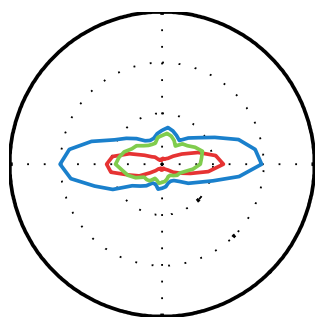

Direction

Figure 3. Saccades in SWJs compared with other small saccades (up to $5^{\circ}$ in size) not in SWJs. Top row, Saccadic peak velocity/ magnitude relationships for the three subject groups. Saccades in SWJs and saccades not in SWJs follow the same peak velocity/ magnitude relationship in each subject group. The slope of the main sequence linear fit is clearly lower for the PSP patients than for the control groups, indicating slower saccades. Middle row, Saccade magnitude distributions for the three subject groups. Ninetysix percent of all saccades in the control groups are $<1^{\circ}$ in size. In contrast, the PSP group exhibits a wide distribution of saccade magnitudes, up to $3-4^{\circ}$. Bottom row, Polar histograms of saccade directions for the three subject groups. Saccades in SWJs are exclusively horizontal for the three groups. Only the control groups show a significant amount of vertical saccades (not in SWJs).

than inter-SWJ ISIs. However, the intra-SWJ ISIs were equivalent for patients and controls (Fig. 4D, Table 1), consistent with the idea that the same coupling mechanism is responsible for SWJs in PSP patients and in healthy subjects.

A number of studies have proposed that an enlargement of horizontal fixational saccades (microsaccades) produces squarewave coupling in healthy subjects (Ditchburn and Ginsborg, 1953; Vedel-Jensen, 1966; Yamazaki, 1968; Feldon and Langston,
1977; Gowen et al., 2007). To confirm this possibility, we calculated the likelihood of a saccade being part of a SWJ as a function of its magnitude. Figure $4 E$ shows that microsaccade magnitude is positively correlated with SWJ coupling and that the correlation is similar for PSP patients and controls. To our knowledge, this is the first systematic study of the relationship between magnitude of fixational saccades and SWJ coupling, and it shows that a large fixational saccade is usually followed by a second corrective saccade, thus resulting in a SWJ (supplemental Fig. 2, available at www.jneurosci.org as supplemental material). Finally, we found a correlation between saccade magnitude and (lack of) vertical component (Fig. 4F). Saccades tended to be more horizontal as their magnitude increased, especially in the PSP group. Figure $4 G$ exemplifies SWJ coupling for large but not small saccades in one subject.

\section{Differentiation of PSP patients and healthy controls based on saccade and SWJ properties}

The SWJ detecting algorithm can be used to automatically extract and compare several SWJ properties between PSP patients and control subjects: rate, magnitude, peak velocity, size of vertical component, ISI, difference in the direction between first and second saccade, and percentage of saccades that are part of SWJs. Table 1 summarizes comparisons between PSP patients and control subjects (the population values were consistent with those of individual subjects) (supplemental Fig. 3 and supplemental Table 2, available at www.jneurosci.org as supplemental material). Both the rate and magnitude of SWJs were significantly greater in PSP patients than in control subjects. The average peak velocity of saccades in SWJs was also greater in PSP patients than in controls, reflecting their larger size. However, the vertical component of SWJs was smaller in PSP patients than in controls. The average ISI between the first and the second saccade of a SWJ did not differ between PSP patients and controls. The difference in direction between the first and the 
second saccades of SWJs was less variable in PSP patients than in control subjects. Finally, the percentage of saccades that formed SWJs was larger in PSP patients than in controls. The vertical component of non-SWJ saccades was much smaller in PSP than controls. The interval between non-SWJ saccades was decreased in PSP. When all saccades were considered ( Table 1 , right column), PSP patients made more frequent, larger, and faster movements, with smaller vertical components, and shorter ISIs.

We used ROC analyses to determine whether the various SWJ and saccade properties tested above could be used to discriminate across subject populations. The area under the ROC curve serves as a measure of the performance of each property as a discriminator (Green and Swets, 1966; Swets, 1988; Zweig and Campbell, 1993) (Fig. 5). Except for the intra-SWJ ISI, all SWJ properties performed significantly better than chance when discriminating between patients and healthy controls. However, the property that performed best as a discriminator was the average vertical component of non-SWJ saccades. ROC analysis also offered thresholds for each of the properties tested (the optimal working point), which can be used as an indicator of whether a given subject suffers from PSP or not (for details, see Materials and Methods).

It is interesting to note that ISI performed better than chance (both in the ROC analysis and in the two-tailed $t$ test) only when we considered the intervals between non-SWJ saccades, or between all saccades (both inside and outside SWJs), but not between SWJ saccades (i.e., the intra-SWJ ISIs). Thus, it may be that an increase in SWJ rate results in shorter inter-SWJ ISIs, whereas intra-SWJ ISIs stay constant. If so, the intra-SWJ ISI may be an intrinsic characteristic of SWJs and thus related to the neural circuit responsible for the coupling of large fixational saccades.

\section{Discussion}

We set out to determine whether fixational saccades and SWJs made by PSP patients showed different features from those made by controls. We confirmed previous reports that SWJs are larger and more frequent in PSP but also found a more distinctive characteristic: all saccades (inside and outside SWJs) showed markedly smaller vertical components in PSP patients than in controls. Our findings are consistent with the idea that all fixational saccades (including both microsaccades and SI) are essentially the same phenomena. Because large saccades are more likely to be coupled in a SWJ, the higher rate of SWJs in PSP may be the direct result of a pathological increase in fixational saccade magnitudes in these patients. First, we compare properties of SWJs in control subjects and PSP patients. Second, we describe differences between microsaccades and
SWJs in both groups. Third, we discuss the possible substrate for SWJs and their clinical significance.

\section{Comparison of SWJs in PSP patients and control subjects}

SWJs made by PSP patients were larger and more frequent than SWJs made by controls. However, our patients had an established diagnosis of PSP, with a median duration of 4 years, so it remains to be proven whether SWJs made by patients earlier in their disease course are distinguishable from SWJs made by healthy subjects. Future research should moreover establish whether SWJs made by patients with other parkinsonian disorders, such as multiple system atrophy (Rascol et al., 1991; Pinnock et al., 2010), or cerebellar conditions such as Friedreich's ataxia, can be distinguished on this basis. 


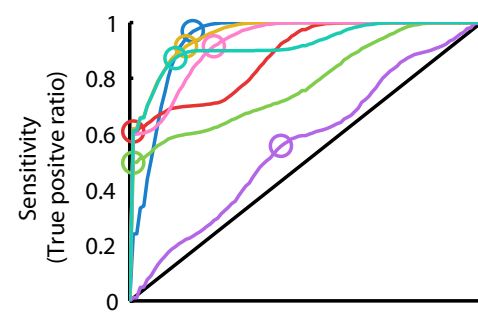

\begin{tabular}{|c|c|}
\hline Property & Threshold \\
\hline SWJ Rate & $>0.3 \mathrm{~N} / \mathrm{s}$ \\
\hline SWJ Avg.Magnitude & $>0.5 \mathrm{deg}$ \\
\hline SWJ Avg.Peak Velocity & $>40 \mathrm{deg} / \mathrm{s}$ \\
\hline SWJ Avg.Vertical Component & $<0.2$ \\
\hline Intra-SWJAvg.ISI & ---------- \\
\hline Std. ofDirection Difference & $<20$ deg \\
\hline Percent of Saccadesin SWJs & $>50 \%$ \\
\hline Non-SWJ Saccade Rate & - ------- \\
\hline Non-SWJ Saccade Avg. Mag nitude & $>0.5 \mathrm{deg}$ \\
\hline Non-SWJ Saccade Avg.Peak Velocity & $>30 \mathrm{deg} / \mathrm{s}$ \\
\hline Non-SWJ Saccade Avg. Vertical Component & $<0.5$ \\
\hline Non-SWJ Saccade ISI & $<1000 \mathrm{~ms}$ \\
\hline Saccade Rate & $>1.2 \mathrm{~N} / \mathrm{s}$ \\
\hline Saccade Avg. Mag nitude & $>0.6 \mathrm{deg}$ \\
\hline Saccade Avg. Peak Velocity & $>35 \mathrm{deg} / \mathrm{s}$ \\
\hline Saccade Avg.Vertical Component & $<0.4$ \\
\hline SaccadeISI & $<600 \mathrm{~ms}$ \\
\hline
\end{tabular}
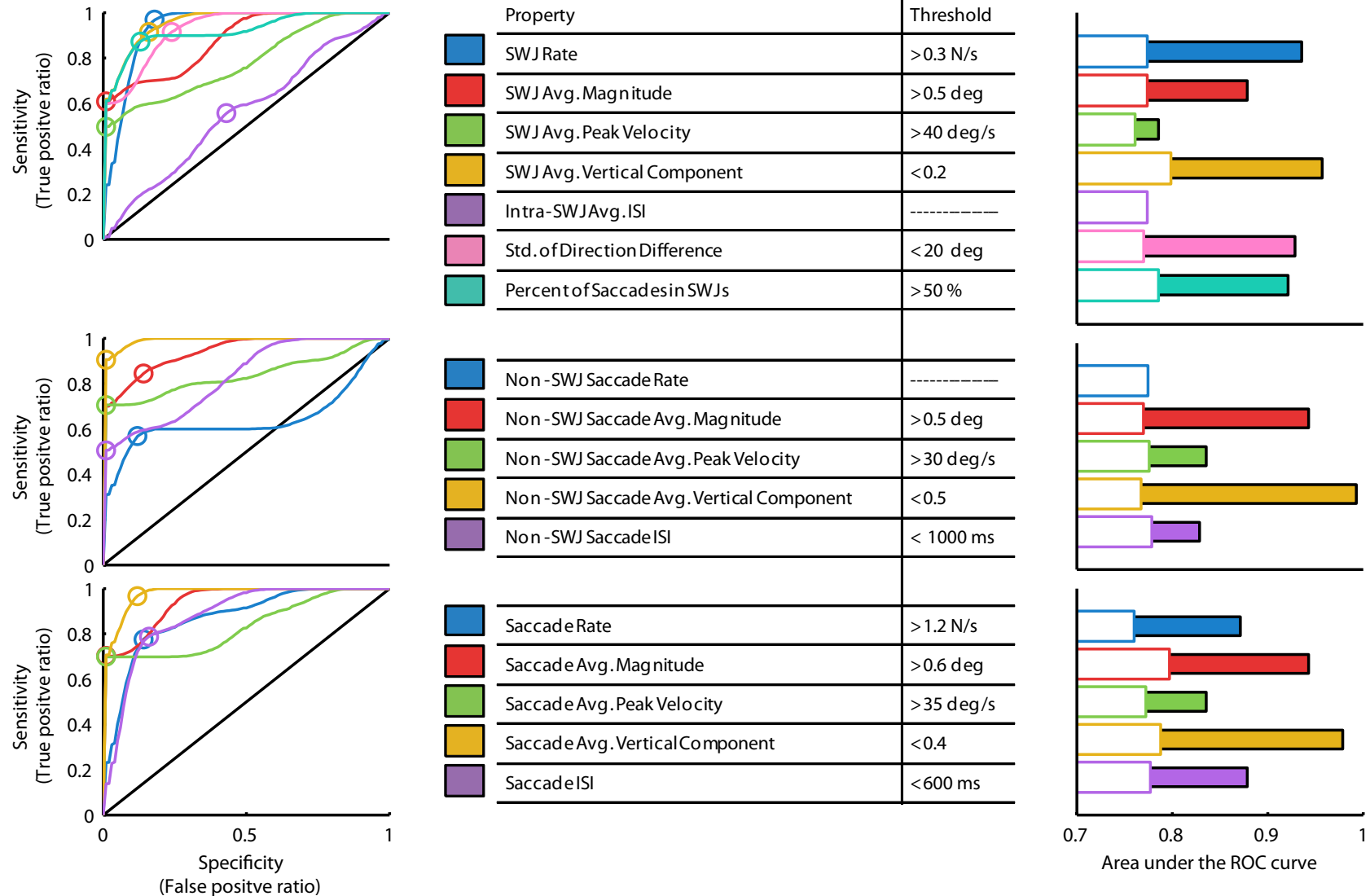

Figure 5. ROC analysis. We used ROC analyses to evaluate the performance of the different SWJ and saccade properties as a test to discriminate between the PSP and the control populations. The area under the ROC curve serves a measurement of this performance. Left column, ROC curves for the different properties, for SWJs (top), non-SWJ saccades (middle), and all saccades (bottom). The optimal working point in each curve is represented with a circle and the corresponding value (threshold) appears in the inset table. Middle column, List of SWJ and saccade properties tested, with respective thresholds. Right column, Area under the ROC curve for each SWJ and saccade property. The solid bars represent the area under the curve; the empty bars indicate the level necessary to reach significance ( 0.01 level) over chance (determined by permutation analysis). To calculate the vertical component of saccades of different sizes, we first normalized the magnitude of all saccades to $1^{\circ}$.

Such studies seem justified (Rascol et al., 1991; Garbutt et al., 2004; Antoniades et al., 2007; Pinnock et al., 2010). In our analyses, the most powerful discriminator, evident during ROC analysis (Fig. 5), was the size of vertical components of saccades.

\section{The relationship between SWJs and microsaccades}

Are microsaccades and SWJs part of a continuum? In other words, are microsaccades and SWJs "alternate descriptions for the same underlying phenomena" (Gowen et al., 2007)? Gowen et al. (2007) have argued that the distinction between microsaccades and SIs is fundamentally methodological: low-resolution eye-trackers in early SI studies may have contributed to the larger magnitudes and smaller rates found in SIs versus microsaccades. Additionally, SI studies usually set minimum magnitude thresholds to $\geq 0.5^{\circ}$, thus arbitrarily excluding many small fixational saccades (Herishanu and Sharpe, 1981; Shallo-Hoffmann et al., 1989, 1990; Salman et al., 2008). Furthermore, SIs studies typically count the first and second (return) saccade as a single SI, whereas most microsaccade studies count the return saccade as a separate microsaccade.

The proposal that microsaccades and SIs are essentially the same phenomenon with two different names is supported by their common peak velocity relationship (Fig. 3, top row) and their similar modulation by attention and task instructions (Hafed and Clark, 2002; Gowen et al., 2005). Both microsaccades and SWJs can be voluntarily and transiently suppressed during strict fixation (Ciuffreda et al., 1979; Herishanu and Sharpe,
1981), and we show here for the first time that both SWJs and microsaccades counteract visual fading during fixation (supplemental Fig. 4, available at www.jneurosci.org as supplemental material).

Other evidence points to dissimilarities between microsaccades and SWJs, however. First, even when SWJs and microsaccades are measured with the same exact methods (as in the current study), SWJs saccades tend to be larger than microsaccades. Second, intra-SWJ ISIs vary little in comparison with the more variable ISIs of microsaccades (Engbert and Kliegl, 2004; Engbert and Mergenthaler, 2006). These apparent differences can be explained by two complementary mechanisms: one to generate fixational saccades and another to produce SWJs when the first saccade is too large (Fig. 4C). Finally, healthy subjects produce vertical and oblique saccades outside of SWJs, and mainly horizontal saccades inside SWJs (Fig. 3, bottom row), perhaps because fixation saccades become more horizontal as their magnitude increases (Fig. 4D).

Our results support the idea of a continuum between microsaccades and SIs (or at least between microsaccades and SWJs). Future studies should investigate the relationship between microsaccades and other types of saccadic intrusions.

\section{Possible neural substrate for saccades during attempted fixation}

Behavioral evidence from human studies supports a common neural mechanism for the generation of saccades and microsac- 
cades. Otero-Millan et al. (2008) found equivalent ISI distributions for exploratory saccades and microsaccades. Rolfs et al. $(2006,2008 \mathrm{a})$ found that microsaccades interact temporally with subsequent large saccades made to a target and proposed a common coding of microsaccades and saccades in the superior colliculus (SC) motor map. Gowen et al. (2007) found that exogenous and endogenous attention modulate both SIs and microsaccades, also supporting a common involvement of the SC. The predictions from the above studies were validated by Hafed et al. (2009), who recently showed that the rostral pole of the SC is active before and during microsaccades. Van Gisbergen and coworkers (Van Gisbergen and Robinson, 1977; Van Gisbergen et al., 1981) had previously found that burst neurons in the brainstem are active during saccades and microsaccades, and Brien et al. (2009) recently showed modulations in the activity of omnipause neurons during microsaccade generation. Similar microsaccade-generating circuits may be at play in other vertebrate species. For instance, Delgado-Garcia et al. (1986) recorded extraocular motoneuronal activity during some putative microsaccades in the alert cat. (For a comparative review of the known dynamics, physiology, and perceptual effects of fixational eye movements across vertebrates, see Martinez-Conde and Macknik, 2008.)

SWJs occur in most people and are especially prevalent in patients suffering from various neurological conditions that affect the cerebral hemispheres (Sharpe et al., 1982), the basal ganglia (Rascol et al., 1991), or the cerebellum (Dale et al., 1978). Elidan et al. (1984) stated that "SWJ are not necessarily associated with CNS pathology and may be a normal phenomenon... a pathological increase of the ocular fixation movements normally present ... rather than evidence of a localized lesion in the CNS." If this idea is correct, any enlargement of fixational saccades, whether pathological or not, should lead to SWJs.

Our results support the notion that SWJs result from a normal mechanism (common to patients and healthy subjects) that couples large fixational saccades with return saccades. PSP patients and control subjects had equivalent intra-SWJ ISIs (Table 1) and showed similar increases in the likelihood of SWJs after large saccades (Fig. 4C). SWJ coupling follows from current models of microsaccade generation, in that the error created by the initial microsaccade could trigger a corrective saccade (Cornsweet, 1956; Martinez-Conde et al., 2004, 2009; Rolfs et al., 2008b; Hafed et al., 2009; Rolfs, 2009). Our present findings do not provide an indication as to whether SWJ coupling might result from an error signal in the SC, the cerebellum, and/or a different structure. Future studies should determine whether patients affected with other neurological conditions have the same intraSWJ ISIs and size-dependent saccadic coupling as PSP patients.

What produces the frequent, large SWJs in the PSP patients? First, it may represent a response to combat the increased risks of sensory adaptation consequent on voluntary gaze palsy in PSP. Second, SJWs could be an adaptive mechanism to promote (nonfixational) vertical saccades; the latter are speeded up when they occur synchronously with SWJs (Garbutt et al., 2004). A third possibility is that frequent SWJs in PSP may be attributable to changes in the cerebellum and its output through the superior cerebellar peduncle, both of which are affected in PSP (Kanazawa et al., 2009; Dickson et al., 2010). Thus, SWJs are prominent in certain cerebellar disorders, such as Friedreich's ataxia (Fahey et al., 2008), in which vertical saccades may be of normal speed and the midbrain is spared. The mechanisms for prominent SWJ in some cerebellar disorders is unclear but might reflect a disturbance of the mechanism by which cortical Purkinje cells suppress visual prompts to make a saccade at the level of the fastigial nuclei (Serra et al., 2008). Fourth, frequent, large SWJs in PSP might be, as noted above, attributable to an enlargement of fixation saccades because of impaired inhibition of the SC by the substantia nigra pars reticulata. Future studies will aim to find a model of the SWJ coupling mechanism and the abnormal saccade magnitudes found in the different diseases.

\section{Conclusions}

We have developed analytic tools to objectively identify and characterize SWJs and found clear differences between the size and frequency of SWJs made by healthy subjects and patients with PSP. However, the most distinctive characteristic is that small saccades lose their vertical component in PSP, and this property may help clinicians differentiate PSP from other parkinsonian or cerebellar disorders that show involuntary saccades during attempted fixation. We have also shown that as microsaccades become larger they are more likely to be followed by a corrective saccade, forming a SWJ. Finally, PSP patients and controls showed equivalent intra-SWJ ISIs, indicating a common neural substrate for the generation of normal and pathological SWJs. We conclude that microsaccades and SIs are essentially the same phenomena and that SWJs are generated by a common coupling mechanism in PSP patients and healthy observers.

\section{References}

Abadi RV, Gowen E (2004) Characteristics of saccadic intrusions. Vision Res 44:2675-2690.

Antoniades CA, Bak TH, Carpenter RH, Hodges JR, Barker RA (2007) Diagnostic potential of saccadometry in progressive supranuclear palsy. Biomark Med 1:487-490.

Brien DC, Corneil BD, Fecteau JH, Bell AH, Munoz DP (2009) The behavioural and neurophysiological modulation of microsaccades in monkeys. J Eye Mov Res 3:1-12.

Britten KH, Shadlen MN, Newsome WT, Movshon JA (1992) The analysis of visual motion: a comparison of neuronal and psychophysical performance. J Neurosci 12:4745-4765.

Ciuffreda KJ, Kenyon RV, Stark L (1979) Suppression of fixational saccades in strabismic and anisometropic amblyopia. Ophthalmic Res 11:31-39.

Cornsweet TN (1956) Determination of the stimuli for involuntary drifts and saccadic eye movements. J Opt Soc Am 46:987-993.

Dale RT, Kirby AW, Jampel RS (1978) Square wave jerks in Friedreich's ataxia. Am J Ophthalmol 85:400-406.

Delgado-Garcia JM, del Pozo F, Baker R (1986) Behavior of neurons in the abducens nucleus of the alert cat- $\mathrm{I}$. Motoneurons. Neuroscience 17:929-952.

Dickson DW, Ahmed Z, Algom AA, Tsuboi Y, Josephs KA (2010) Neuropathology of variants of progressive supranuclear palsy. Curr Opin Neurol 23:394-400.

Ditchburn RW, Ginsborg BL (1953) Involuntary eye movements during fixation. J Physiol 119:1-17.

Elidan J, Gay I, Lev S (1984) Square wave jerks-incidence, characteristic, and significance. J Otolaryngol 13:375-381.

Engbert R (2006) Microsaccades: a microcosm for research on oculomotor control, attention, and visual perception. Prog Brain Res 154:177-192.

Engbert R, Kliegl R (2003) Microsaccades uncover the orientation of covert attention. Vision Res 43:1035-1045.

Engbert R, Kliegl R (2004) Microsaccades keep the eyes' balance during fixation. Psychol Sci 15:431-436.

Engbert R, Mergenthaler K (2006) Microsaccades are triggered by low retinal image slip. Proc Natl Acad Sci U S A 103:7192-7197.

Evinger C, Shaw MD, Peck CK, Manning KA, Baker R (1984) Blinking and associated eye movements in humans, guinea pigs, and rabbits. J Neurophysiol 52:323-339.

Fahey MC, Cremer PD, Aw ST, Millist L, Todd MJ, White OB, Halmagyi M, Corben LA, Collins V, Churchyard AJ, Tan K, Kowal L, Delatycki MB (2008) Vestibular, saccadic and fixation abnormalities in genetically confirmed Friedreich ataxia. Brain 131:1035-1045.

Feierstein CE, Quirk MC, Uchida N, Sosulski DL, Mainen ZF (2006) 
Representation of spatial goals in rat orbitofrontal cortex. Neuron 51:495-507.

Feldon SE, Langston JW (1977) Square-wave jerks: a disorder of microsaccades? Neurology 27:278-281.

Garbutt S, Riley DE, Kumar AN, Han Y, Harwood MR, Leigh RJ (2004) Abnormalities of optokinetic nystagmus in progressive supranuclear palsy. J Neurol Neurosurg Psychiatry 75:1386-1394.

Gowen E, Abadi RV, Poliakoff E (2005) Paying attention to saccadic intrusions. Brain Res Cogn Brain Res 25:810-825.

Gowen E, Abadi RV, Poliakoff E, Hansen PC, Miall RC (2007) Modulation of saccadic intrusions by exogenous and endogenous attention. Brain Res 1141:154-167.

Green DM, Swets JA (1966) Signal detection theory and psychophysics. New York: Wiley.

Gruart A, Blázquez P, Delgado-García JM (1995) Kinematics of spontaneous, reflex, and conditioned eyelid movements in the alert cat. J Neurophysiol 74:226-248.

Hafed ZM, Clark JJ (2002) Microsaccades as an overt measure of covert attention shifts. Vision Res 42:2533-2545.

Hafed ZM, Goffart L, Krauzlis RJ (2009) A neural mechanism for microsaccade generation in the primate superior colliculus. Science 323:940-943.

Herishanu YO, Sharpe JA (1981) Normal square wave jerks. Invest Ophthalmol Vis Sci 20:268-272.

Hernández A, Zainos A, Romo R (2002) Temporal evolution of a decisionmaking process in medial premotor cortex. Neuron 33:959-972.

Kanazawa M, Shimohata T, Toyoshima Y, Tada M, Kakita A, Morita T, Ozawa T, Takahashi H, Nishizawa M (2009) Cerebellar involvement in progressive supranuclear palsy: a clinicopathological study. Mov Disord 24:1312-1318.

Kapoula ZA, Robinson DA, Hain TC (1986) Motion of the eye immediately after a saccade. Exp Brain Res 61:386-394.

Laubrock J, Engbert R, Kliegl R (2005) Microsaccade dynamics during covert attention. Vision Res 45:721-730.

Leigh RJ, Zee DS (2006) The neurology of eye movements, Ed 4. Oxford: Oxford UP.

Litvan I, Agid Y, Calne D, Campbell G, Dubois B, Duvoisin RC, Goetz CG, Golbe LI, Grafman J, Growdon JH, Hallett M, Jankovic J, Quinn NP, Tolosa E, Zee DS (1996) Clinical research criteria for the diagnosis of progressive supranuclear palsy (Steele-Richardson-Olszewski syndrome): report of the NINDS-SPSP international workshop. Neurology $47: 1-9$.

Martinez-Conde S (2006) Fixational eye movements in normal and pathological vision. Prog Brain Res 154:151-176.

Martinez-Conde S, Macknik SL (2008) Fixational eye movements across vertebrates: comparative dynamics, physiology, and perception. J Vis 8:28.1-16.

Martinez-Conde S, Macknik SL, Hubel DH (2000) Microsaccadic eye movements and firing of single cells in the striate cortex of macaque monkeys. Nat Neurosci 3:251-258.

Martinez-Conde S, Macknik SL, Hubel DH (2002) The function of bursts of spikes during visual fixation in the awake primate lateral geniculate nucleus and primary visual cortex. Proc Natl Acad Sci U S A 99:13920-13925.

Martinez-Conde S, Macknik SL, Hubel DH (2004) The role of fixational eye movements in visual perception. Nat Rev Neurosci 5:229-240.

Martinez-Conde S, Macknik SL, Troncoso XG, Hubel DH (2009) Microsaccades: a neurophysiological analysis. Trends Neurosci 32:463-475.

Møller F, Laursen ML, Tygesen J, Sjølie AK (2002) Binocular quantification and characterization of microsaccades. Graefes Arch Clin Exp Ophthalmol 240:765-770.

Otero-Millan J, Troncoso X, Macknik SL, Serrano-Pedraza I, MartinezConde S (2008) Saccades and microsaccades during visual fixation, exploration and search: foundations for a common saccadic generator. J Vis 8:21.1-18.
Pinnock RA, McGivern RC, Forbes R, Gibson JM (2010) An exploration of ocular fixation in Parkinson's disease, multiple system atrophy and progressive supranuclear palsy. J Neurol 257:533-539.

Ramat S, Somers JT, Das VE, Leigh RJ (1999) Conjugate ocular oscillations during shifts of the direction and depth of visual fixation. Invest Ophthalmol Vis Sci 40:1681-1686.

Rascol O, Sabatini U, Simonetta-Moreau M, Montastruc JL, Rascol A, Clanet M (1991) Square wave jerks in parkinsonian syndromes. J Neurol Neurosurg Psychiatry 54:599-602.

Robinson DA (1963) A method of measuring eye movement using a scleral search coil in a magnetic field. IEEE Trans Biomed Electron BME 10:137-145.

Rolfs M (2009) Microsaccades: small steps on a long way. Vision Res 49:2415-2441.

Rolfs M, Laubrock J, Kliegl R (2006) Shortening and prolongation of saccade latencies following microsaccades. Exp Brain Res 169:369-376.

Rolfs M, Laubrock J, Kliegl R (2008a) Microsaccade-induced prolongation of saccadic latencies depends on microsaccade amplitude. J Eye Mov Res $1: 1-8$.

Rolfs M, Kliegl R, Engbert K (2008b) Toward a model of microsaccade generation: the case of microsaccadic inhibition. J Vis 8:1-23.

Romo R, Hernández A, Zainos A (2004) Neuronal correlates of a perceptual decision in ventral premotor cortex. Neuron 41:165-173.

Salman MS, Sharpe JA, Lillakas L, Steinbach MJ (2008) Square wave jerks in children and adolescents. Pediatr Neurol 38:16-19.

Serra A, Liao K, Martinez-Conde S, Optican LM, Leigh RJ (2008) Suppression of saccadic intrusions in hereditary ataxia by memantine. Neurology 70:810-812.

Shallo-Hoffmann J, Petersen J, Mühlendyck H (1989) How normal are "normal" square wave jerks? Invest Ophthalmol Vis Sci 30:1009-1011.

Shallo-Hoffmann J, Sendler B, Mühlendyck H (1990) Normal square wave jerks in differing age groups. Invest Ophthalmol Vis Sci 31:1649-1652.

Sharpe JA, Fletcher WA (1986) Disorders of visual fixation. In: Neuroophthalmology now! (Lawton Smith J, ed), pp 267-284. Chicago: Field, Rich, and Associates.

Sharpe JA, Herishanu YO, White OB (1982) Cerebral square wave jerks. Neurology 32:57-62.

Swets JA (1988) Measuring the accuracy of diagnostic systems. Science 240:1285-1293.

Troncoso XG, Macknik SL, Otero-Millan J, Martinez-Conde S (2008a) Microsaccades drive illusory motion in the Enigma illusion. Proc Natl Acad Sci U S A 105:16033-16038.

Troncoso X, Macknik SL, Martinez-Conde S (2008b) Microsaccades counteract perceptual filling-in. J Vis 8:15.1-9.

Troost BT, Daroff RB (1977) The ocular motor defects in progressive supranuclear palsy. Ann Neurol 2:397-403.

Van Gisbergen JA, Robinson DA, Gielen S (1981) A quantitative analysis of generation of saccadic eye movements by burst neurons. J Neurophysiol 45:417-442.

Van Gisbergen JAM, Robinson DA (1977) Generation of micro and macrosaccades by burst neurons in the monkey. In: Control of gaze by brain stem neurons (Baker R, Berthoz A, eds). New York: Elsevier/North-Holland.

Vedel-Jensen N (1966) Pathological ocular fixation instability. (Photonystagmographic investigations of ocular fixation movements in patients suffering from the post-traumatic cerebral syndrome, and disseminated sclerosis, compared to normal subjects). Acta Ophthalmol (Copenh) 44:481-493.

Yamazaki A (1968) Electrophysiological study on "flick" eye movements during fixation (in Japanese). Nippon Ganka Gakkai Zasshi 72:2446-2459.

Zweig MH, Campbell G (1993) Receiver-operating characteristic (ROC) plots: a fundamental evaluation tool in clinical medicine. Clin Chem 39:561-577. 